\title{
Programming Mental Health: Risk from Adverse Experience for Mothers and Offspring
}

\author{
Alison J. Douglas \\ Centre for Integrative Physiology, University of Edinburgh, Edinburgh, UK
}

Environmental conditions have long been known to affect behaviour, mood, mental state and cognition in adults and children. Likewise, exposure to stress during pregnancy and its long-term adverse effects on fetal programming are becoming well understood, especially for consequences such as cardiovascular disease, obesity and depression [1]. However, what is now emerging is an appreciation that programming of mental health also occurs, and that it can be initiated during gestation, having profound harmful effects on both the maternal and offspring brains [2].

International expertise in parental and offspring neurophysiology was brought together at The Parental Brain IV: Neurobiology Behaviour and the Next Generation, held in Edinburgh on September 1-4, 2010. The local organising committee was chaired by Alison Douglas and John Russell and included Simone Meddle, Megan Holmes and Paula Brunton, supported by a broad International Scientific Advisory Board representing all the major parental brain research groups around the world. This special issue of Neuroendocrinology gathers together the conference presentations on Perinatal Influence on Mental Health in the format of timely reviews. This was one of the four main themes of the conference, which attracted 24 symposium speakers and 3 plenary lectures; there were also 16 oral communications and 23 datablitz pre- sentations. Other journals are publishing papers from each of the other symposia: Stress will publish papers from the Early Life Programming symposium, and the Journal of Neuroendocrinology will publish papers from the Maternal Adaptations in Pregnancy and Lactation and Parental Behaviour symposia.

The papers in this issue give a current picture of the field of programming of mental health research, addressing the long-term effects of suboptimal environmental conditions on both the mother and her offspring. For the first time, recent studies in women have revealed that the mother is susceptible to programming of her behaviour and mental health during gestation (see Sandman et al., this issue). Pregnancy stress and anxiety alter her cognitive performance, memory and increase her risk of altered maternal caring ability and postpartum depression after birth. Indeed, quality of maternal behaviour is even dependent upon whether the mother gave birth vaginally or by caesarean section [3]. These changes in mental state, along with other physiological (e.g. neuroendocrine) changes, directly and indirectly impact on the developing embryo/fetus and later the neonate. This can lead to altered behaviour and mental health of the offspring both during pregnancy (in parallel with the mother) and for many years following birth (Sandman et al.).

\section{KARGER}

Fax +4161306 1234 E-Mail karger@karger.ch www.karger.com
(C) 2012 S. Karger AG, Basel

0028-3835/12/0951-0005\$38.00/0

Accessible online at:

www.karger.com/nen
Alison J. Douglas

Centre for Integrative Physiology, University of Edinburgh

Hugh Robson Building, George Square

Edinburgh EH8 9XD (UK)

Tel. +44 131650 3274, E-Mail alison.j.douglas@ed.ac.uk 


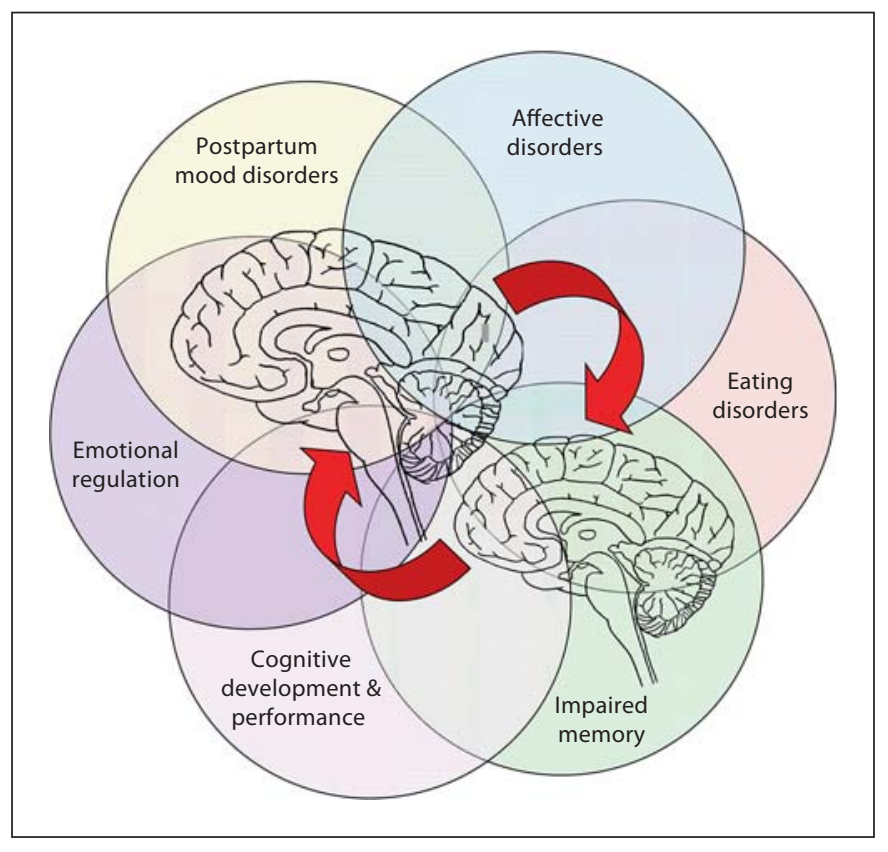

Fig. 1. Schematic indicating potential mental health problems that can arise in mother and offspring from exposure to adverse environmental conditions. The mother's responses can impact profoundly on the baby directly and indirectly and, vice versa, the baby's responses influence the mother.

Changes in maternal mental health in pregnancy and perinatally are common; depression and pregnancy anxiety often emerge. Classical perinatal mental health issues that have been extensively investigated are postpartum blues, postpartum anxiety and the related postnatal depression (and the more serious, postpartum psychosis). The extensive hormone changes that occur throughout pregnancy and at and after birth, combined with physical exhaustion, and predisposition to depression can trigger these conditions in new mothers. It is normal for the mother's brain to undergo adaptations in many brain networks during pregnancy, and these prepare her for birth and lactation and their associated behaviours. However, a new take on the underlying cause of stress-induced mental health changes is that the stress alters or prevents the normal adaptation of the mother's brain and neuroendocrine systems, including responses to stress. Studies have shown that chronic stress during gestation can prevent the normal maternal brain adaptations and so can increase the risk of the above perinatal mood disorders (reviewed in Hillerer et al., this issue). Therefore, it seems that the extensive adaptations are necessary, preventing inappropriate maternal mental conditions and without them the mother is susceptible to mental health problems.

Women experiencing mood disorders in pregnancy and postpartum are typically treated with antidepressant drugs such as selective serotonin reuptake inhibitors (SSRI), although often depressive symptoms, while attenuated, continue. However, SSRI cross the placenta and blood-brain barrier to access the fetal brain and as serotonin is directly involved in neurodevelopment there is current realisation that the drugs impact adversely on the offspring. New clinical and preclinical research is probing this concern - initial indications point towards biphasic actions, including part protection from the effects of maternal stress and depression on fetal brain neurodevelopmental markers but also potential long-term impact on offspring neurobehaviour (Pawluski, this issue). This may even be the case following birth as SSRI are present in breast milk. Therefore, together with direct effects of maternal stress on the developing offspring, treatments designed to alleviate maternal symptoms have an additional role to play in determining appropriate neurodevelopment. Furthermore, programming of mental disorders such as anxiety and depressive-like symptoms in mouse models are associated with glucocorticoid-induced changes in serotonin and dopamine systems in the adult brain (Wyrwoll and Holmes, this issue), so clearly adaptations in brain monoamine systems contribute to perinatal influences on mental health.

While both prenatal and neonatal stress exposure effects on offspring physiology and mental health have been studied in depth as reported here, another vulnerable time of offspring development has lately been identified. During childhood the brain is still susceptible to adverse conditions, and trauma or chronic stress during this time can have additional unwanted long-term effects, particularly on mental state. This has been reviewed by Richter-Levin's group (Horovitz et al., in this issue). A useful model has been developed in juvenile rats, and shows dysfunctional cognitive processes, altered cortical activity and susceptibility to augmented responses to stress exposure again later when adult. Underlying mechanisms have been investigated and include changes in maturation of neuronal connectivity and in transmitter release and action. This vulnerability of childhood seems to extend into adolescence since mild stress at puberty in rats can also alter behaviour. Although depressive-like behaviour is not influenced, risk-taking behaviour is, evidently enhancing independence-building activities [4].

So, in common with the gestational stress consequences, the adaptations of the mother and offspring at differ- 
ent stages likely adjust the brain to coping optimally with the same type of environment in the future. Common themes emerge from the reviews in this issue. Stress or adverse environment have detrimental effects on mental state, regardless of when during the lifetime they are experienced (i.e., as an embryo/fetus/neonate/juvenile/adolescent or adult). They depend upon alteration or prevention of the normal brain adaptations that underlie optimal physiology and mental health, and so during times of development or change (such as pregnancy and lactation, but also other periods) are particularly susceptible. Reciprocal exchange of signals between the mother and fetus (e.g. hormonal via the placenta), or mother and neonate (e.g. neuronal via suckling) can facilitate (or compromise) appropriate (inappropriate) adaptations of both their brains (fig. 1). It is clear that susceptibility is genderrelated: females are particularly vulnerable to unstable mental health due to monthly reproductive cyclic changes in the brain and body, as well as the stages of pregnancy and lactation. However, these physiological conditions alone are not enough to cause mental disorders, which rely on the additional exposure to triggers, whether physical, social or environmental. Finally, as it is known that the epigenetic influence on fetal programming of stress responsiveness can be passed on from one generation to another, the question now arises whether programming of mental health can also be epigenetically inherited? The finding that mother and baby simultaneously respond to stress during gestation (Sandman et al.) suggests that it can. The underlying mechanisms of environmental influence on mental state are now being elucidated by the laboratories these reviews emanate from, and the future promises further deep insight into understanding and treatment of mental health.

\section{References}

Programming Mental Health

\footnotetext{
1 Weinstock M: The long-term behavioura consequences of prenatal stress. Biobehav Rev 2008;32:1073-1086.

2 Glynn LM, Sandman CA: The influence of prenatal stress and adverse birth outcomes on human cognitive and neurological development; in International Review of Research in Mental Retardation. New York, Academic Press, 2006, pp 109-129.
}

-3 Swain JE, Tasgin E, Mayes LC, Feldman R, Constable RT, Leckman JF: Maternal brain response to own baby-cry is affected by cesarean section delivery. J Child Psychol Psychiatry 2008;10:1042-1052.

$\checkmark 4$ Toledo-Rodriguez M, Sandi C: Stress during adolescence increases novelty seeking and risk-taking behavior in male and female rats. Front Behav Neurosci 2011;5:17. 